\title{
TOXICIDADE AGUDA DA AMÔNIA NÃO IONIZADA E DO NITRITO EM ALEVINOS DE Cichlasoma facetum (JENYNS, 1842)
}

\author{
Acute toxicity of un ionized ammonia and nitrite in Cichlasoma facetum \\ (Jenyns, 1842) fingerlings
}

Sérgio Renato Noguez Piedras', José Luis Ruas Oliveira², Paulo Roberto Rocha Moraes ${ }^{3}$, Alex Bager ${ }^{4}$

\begin{abstract}
RESUMO
A presença de derivados do nitrogênio na água indica processos biológicos ativos influenciados por poluição orgânica, podendo, em altas concentrações, causar danos fisiológicos e até mortalidade em organismos aquáticos. Com o objetivo de determinar o efeito letal da amônia não ionizada e do nitrito, alevinos de cará (Cichlasoma facetum) foram expostos por 96 horas a diversas concentrações desses compostos: de 0 a 4,60 mg. $\mathrm{L}^{-1}$ de $\mathrm{NH}_{3}-\mathrm{N}$, e de 0 a $6,68 \mathrm{de}$ mg.L $\mathrm{L}^{-1}$ de $\mathrm{NO}_{2}$. A CL $\mathrm{CL}_{50}$ calculada, para 96 horas, foi de 2,95 mg. $\mathrm{L}^{-1}$ de amônia não ionizada. A maior concentração de nitrito utilizada, 6,68 mg.L $\mathrm{L}^{-1}$, causou a mortalidade de 45,63\% dos animais em 96 horas. A análise fatorial da variância da mortalidade em relação à concentração dos agentes mostrou haver interação entre os dois produtos, tendo como consequiência o aumento da mortalidade.
\end{abstract}

Termos para indexação: Nitrito, amônia, toxicidade, Cichlasoma facetum.

\begin{abstract}
The nitrogen is derivates in water presence indicates active biological processes influenced by organic pollution, being able, in high concentrations, to cause physiological damages, even mortality in aquatic organisms. In order to determine the lethal effect of the un- ionized ammonia and of the nitrite, fingerlings of cará (Cichlasoma facetum) have been exposed, during 96 hours, to diverse concentrations of such composites: from 0 to $4.60 \mathrm{mg} \cdot \mathrm{L}^{-1}$ of $\mathrm{NH}_{3}-\mathrm{N}$, and from 0 to $6.68 \mathrm{mg} . \mathrm{L}^{-1}$ of $\mathrm{NO}_{2}$. The calculated $\mathrm{CL}_{50}$, for 96 hours, was $2.95 \mathrm{mg} . \mathrm{L}^{-1}$ of un-ionized ammonia. The highest concentration of nitrite, $6.68 \mathrm{mg} . \mathrm{L}^{-1}$, caused the mortality of $45.63 \%$ of the fingerlings of cará in 96 hours. The factorial analysis of the mortality variance in relation to the concentration of agents, showed the interaction between both products, resulting the increase of mortality of fingerlings of cará.
\end{abstract}

Index terms: Nitrite, ammonia, toxicity, Cichlasoma facetum.

\section{(Recebido para publicação em 11 de agosto de 2005 e aprovado em 9 de maio de 2006)}

\section{INTRODUÇÃO}

O desenvolvimento agrícola e industrial ocorrido nos últimos anos, resultou no aumento de compostos poluentes lançados no ambiente aquático. De acordo com Esteves (1988), o nitrogênio é um dos elementos mais importantes no metabolismo de ecossistemas aquáticos. Esta importância deve-se principalmente a sua participação na formação de proteínas, um dos compostos básicos da biomassa. Quando presente em baixas concentrações, pode atuar como fator limitante na produção primária de ecossistemas aquáticos. A chuva, o material orgânico e inorgânico de origem alóctone e a fixação biológica por bactérias e algas cianofíceas; são as principais fontes naturais de nitrogênio que o ambiente aquático pode ter.

A função fisiológica do nitrogênio é a formação de proteínas, ácidos nucléicos e coenzimas, sem o mesmo não se formarão as paredes celulares e portanto não existirão os organismos (TIAGO, 2000).
Os compostos nitrogenados aparecem sob três formas no ambiente aquático. O nitrato é a principal forma de nitrogênio encontrada nas águas e, quando em elevadas concentrações, pode conduzir a um processo de produção exagerada, denominado de eutrofização. $O$ nitrogênio amoniacal (amônia), é uma substância tóxica não persistente e não cumulativa e que em concentração baixa, não causa nenhum dano fisiológico aos animais; e por último o nitrito, que é uma forma química do nitrogênio normalmente encontrada em quantidades diminutas nas águas superficiais, pois o nitrito é instável na presença do oxigênio, ocorrendo como uma forma intermediária no processo de nitrificação, no qual a amônia é transformada (oxidada) por bactérias para nitrito, e logo para nitrato, em sistemas aquáticos. $\mathrm{O}$ íon nitrito pode ser utilizado pelas plantas como uma fonte de nitrogênio, e sua presença na água indica processos biológicos ativos influenciados por poluição orgânica (GORSEL \& JENSEN, 1999). Segundo

Oceanólogo, Doutor, Professor da Escola de Ciências Ambientais da Universidade Católica de Pelotas - ECAm - Rua Felix da Cunha, 412 - $96.010-000$ Pelotas, RS - sergiopiedras@ibest.com.br

${ }^{2}$ Acadêmico do Curso de Ecologia da Universidade Católica de Pelotas - ECAm - Rua Felix da Cunha, 412 - 96.010-000 - Pelotas, RS.

${ }^{3}$ Oceanólogo, Especialista, Professor da Escola de Ciências Ambientais da Universidade Católica de Pelotas- ECAm - Rua Felix da Cunha, 412 96.010-000 - Pelotas, RS.

${ }^{4}$ Oceanólogo, Doutor, Professor da Escola de Ciências Ambientais da Universidade Católica de Pelotas -ECAm - Rua Felix da Cunha, 412 - $96.010-000$ - Pelotas, RS. 
Arana (1997), a amônia quando dissolvida na água encontra-se em equilíbrio entre as formas ionizada e não ionizada, sendo este equilíbrio influenciado pelo $\mathrm{pH}$, temperatura e salinidade. Alterações destes parâmetros resultaram na variação da concentração das diversas formas de nitrogênio, que podem atingir concentrações tóxicas para os peixes. A amônia difunde-se facilmente através das membranas respiratórias, causando danos ao epitélio branquial e, como conseqüência, dificulta as trocas gasosas entre o animal e a água, desestabilizando o sistema de osmorregulação. $\mathrm{O}$ nitrito em altas concentrações provoca a oxidação do átomo de ferro da molécula da hemoglobina do sangue, convertendo-a em meta-hemoglobina, molécula incapaz de transportar oxigênio, estabelecendo-se um quadro de hipoxia e cianose (DUBOROW et al., 1997).

O Cará Cichlasoma facetum, pertencente à família Cichlidae, é um peixe nativo da região sul do Brasil. No Rio Grande do Sul ocorre no sistema da Laguna dos Patos, sistema lagunar costeiro e sistema do rio Uruguai, sendo um bom representante da ictiofauna gaúcha (KOCH et al., 2000). Alimenta-se de larvas de insetos, crustáceos, pequenos organismos e lodo, interagindo com toda a cadeia alimentar do ambiente em que se encontra, sendo desta forma, um excelente bioindicador ambiental.

Os estudos toxicológicos através de teste de letalidade $\left(\mathrm{CL}_{50}\right)$ são recomendados, como informação básica para o conhecimento de possíveis danos ambientais resultantes do lançamento de efluentes contaminados nos corpos receptores (IBAMA, 1990).

O presente estudo teve como objetivo determinar a toxicidade do nitrito e da amônia não ionizada em alevinos de cará, com 96 horas de exposição.

\section{MATERIAL E MÉTODOS}

O experimento foi realizado na Estação de Piscicultura da UCPel. Os alevinos de Cará foram obtidos através de reprodução e criados na própria Estação. Os testes de toxicidade aguda seguiram as recomendações descritas em CETESB (1991) e IBAMA (1990).

Foram utilizadas 36 unidades experimentais, sendo cada uma delas composta por um aquário com capacidade de 20 litros, com sistema de aeração, controle de temperatura e cinco alevinos. Os animais apresentavam tamanho médio de 4,29 $\pm 0,55$ centímetros de comprimento total e peso médio de 1,56 $\pm 0,3$ gramas. Os tratamentos, em número de 12 , inteiramente casualizados e com três repetições, foram assim constituídos: 02 concentrações de $\mathrm{NH}_{3}-\mathrm{N}$, respectivamente $2,03 \pm 0,21 \mathrm{mg} . \mathrm{L}^{-1} \mathrm{e} 3,28 \pm 0,20 \mathrm{mg} . \mathrm{L}^{-1} ; 05$ concentrações de $\mathrm{NO}_{2}$, respectivamente $3,58 \pm 0,40 \mathrm{mg} . \mathrm{L}^{-1}$, 4,73 $\pm 0,19 \mathrm{mg} . \mathrm{L}^{-1}, 5,61 \pm 0,21 \mathrm{mg} . \mathrm{L}^{-1}, 6,23 \pm 0,20 \mathrm{mg} . \mathrm{L}^{-1}$, e $6,68 \pm 0,19 \mathrm{mg} . \mathrm{L}^{-1}, 04$ tratamentos de amônia não ionizada junto com nitrito, nas seguintes relações respectivas: 2,20 $\pm 0,10 \mathrm{mg} . \mathrm{L}^{-1}+4,90 \pm 0,05 \mathrm{mg} . \mathrm{L}^{-1} ; 2,19 \pm 0,05 \mathrm{mg} . \mathrm{L}^{-1}+$ $5,82 \pm 0,07 \mathrm{mg} . \mathrm{L}^{-1} ; 4,40 \pm 0,30 \mathrm{mg} . \mathrm{L}^{-1}+4,70 \pm 0,10 \mathrm{mg} \cdot \mathrm{L}^{-1} ; \mathrm{e}$ $4,60 \pm 0,07 \mathrm{mg} \cdot \mathrm{L}^{-1}+5,67 \pm 0,18 \mathrm{mg} . \mathrm{L}^{-1} \mathrm{e}$ por último, o controle.

A água dos aquários foi proveniente de uma solução estoque previamente preparada, através da floculação com sulfato de alumínio como medida preventiva para eliminar a presença de nitrogênio orgânico e a correção do $\mathrm{pH}$ efetuada com carbonato de cálcio. A concentração de amônia gasosa foi induzida pela introdução de Cloreto de Amônio e o Nitrito a partir do Nitrato de Sódio (ARANA, 1997).

As variáveis físicas e químicas: temperatura, oxigênio dissolvido, $\mathrm{pH}$, gás carbônico, alcalinidade, condutividade, amônia e nitritos foram monitorados diariamente, segundo APHA (1998). A concentração de amônia não ionizada foi calculada, como percentual da amônia total, considerando-se a temperatura e o pH, pelo método de Nesslerização (APHA, 1998).

$\mathrm{O}$ efeito dos produtos foi observado através da mortalidade ocorrida em cada tratamento a cada 24 horas. Os dados foram tabulados e submetidos à análise de variância (Pd" 0,05), sendo as diferenças entre os tratamentos verificadas pelo teste de Duncan. O comportamento da mortalidade em relação às concentrações de amônia não ionizada e do nitrito é representada por regressão. A ocorrência de interação entre a amônia e o nitrito, foi determinada através da análise fatorial da variância entre todos os tratamentos. Os dados foram processados usando-se o software estatístico SAS Institute (1998).

\section{RESULTADOS E DISCUSSÃO}

Durante o período experimental a água dos aquários apresentou características físicas e químicas em limites considerados aceitaveis para a espécie, sendo que as variações observadas de conditividade, gás carbônico, condutividade, amônia não ionizada e nitrito, foram resultantes da diluíção das soluções de $\mathrm{NH}_{4} \mathrm{Cl}$ e de $\mathrm{NaNO}_{2}$. para produzir as concentrações de amônia não ionizada e de nitrito desejadas.

Na Tabela 1, apresentam-se os resultados médios das principais variáveis avaliadas. A concentração de amônia não ionizada variou entre 2,03 $\pm 0,21$ e 3,28 $\pm 0,20$ mg. $\mathrm{L}^{-1}$ e o nitrito entre $3,58 \pm 0,4$ e $6,68 \pm 0,19 \mathrm{mg} \cdot \mathrm{L}^{-1}$. 
TABELA 1 - Médias de temperatura $\left(\mathrm{T}\right.$ em $\left.{ }^{\circ} \mathrm{C}\right)$, oxigênio dissolvido $\left(\mathrm{O}_{2} \mathrm{em} \mathrm{mg.} \mathrm{L}^{-1}\right)$, saturação de oxigênio dissolvido (Sat.

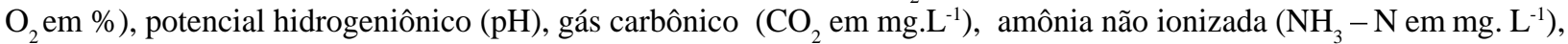
alcalinidade (Alcal. em mg. $\mathrm{L}^{-1}$ de $\mathrm{Ca} \mathrm{CO}_{3}$ ), condutividade (Cond. em Mmohs.cm-1), nitrito $\left(\mathrm{NO}_{2} \mathrm{mg}^{-1} \mathrm{~L}^{-1}\right.$ ) e percentual de mortalidade (M\%) nos tratamentos utilizados de toxicidade aguda da amônia não ionizada e do nitrito em Cará.

\begin{tabular}{lcccccccccc}
\hline Tratamento & $\mathbf{T}^{\mathbf{0}} \mathbf{C}$ & $\mathbf{O}_{\mathbf{2}}$ & $\begin{array}{c}\text { Sat. } \\
\mathbf{O}_{\mathbf{2}}\end{array}$ & $\mathbf{p H}$ & $\mathbf{C O}_{\mathbf{2}}$ & $\begin{array}{c}\mathbf{N H}_{\mathbf{3}}-\mathbf{N} \\
\left(\mathbf{m g .} \mathbf{~}^{-\mathbf{1}}\right)\end{array}$ & Alcal. & $\mathbf{C o n d}$ & $\begin{array}{c}\mathbf{N O}_{\mathbf{2}} \\
\left(\mathbf{m g . L}^{\mathbf{1}} \mathbf{)}\right.\end{array}$ & $\begin{array}{c}\mathbf{M} \\
(\boldsymbol{\%})\end{array}$ \\
\hline T1 & 21,30 & 6,63 & 74 & 7,88 & 2,33 & 0,0 & 49 & 163 & 3,58 & 0 \\
T2 & 20,93 & 6,78 & 71 & 7,91 & 2,16 & 0,0 & 48 & 168 & 4,73 & 0 \\
T3 & 20,93 & 6,45 & 75 & 7,95 & 2,33 & 0,0 & 51 & 181 & 5,61 & $20,0 \mathrm{c}$ \\
T4 & 20,93 & 6,82 & 75 & 7,94 & 2,16 & 0,0 & 51 & 191 & 6,23 & $33,3 \mathrm{c}$ \\
T5 & 20,93 & 6,60 & 71 & 7,94 & 6,66 & 0,0 & 60 & 420 & 6,68 & $60,0 \mathrm{~b}$ \\
T6 & 20,63 & 6,46 & 70 & 7,78 & 10,00 & 2,03 & 59 & 561 & 0,0 & $6,70 \mathrm{~d}$ \\
T7 & 20,73 & 6,82 & 74 & 7,73 & 12,00 & 3,28 & 65 & 746 & 0,0 & $66,70 \mathrm{~b}$ \\
T8 & 21,23 & 6,66 & 75 & 7,77 & 8,66 & 2,20 & 60 & 402 & 4,90 & $13,30 \mathrm{~d}$ \\
T9 & 20,93 & 6,89 & 75 & 7,78 & 9,66 & 2,19 & 59 & 521 & 5,82 & $26,70 \mathrm{c}$ \\
T10 & 21,23 & 6,89 & 74 & 7,78 & 9,66 & 4,40 & 67 & 673 & 4,70 & $100,0 \mathrm{a}$ \\
T11 & 21,33 & 6,87 & 71 & 7,76 & 10,00 & 4,60 & 69 & 417 & 5,67 & $100,0 \mathrm{a}$ \\
T12 & 20,73 & 6,51 & 72 & 7,90 & 6,00 & 0,0 & 70 & 160 & 0,0 & 0,0 \\
D.P & 0,099 & 0,39 & 4,92 & 0,13 & 3,18 & 1,495 & 8,44 & 207,57 & 1,495 & 30,44 \\
\hline
\end{tabular}

Letras diferentes na coluna da mortalidade, indicam, haver diferença significativa pelo teste de Duncan $(\mathrm{P} \leq 0,05)$.

O comportamento da mortalidade em relação a concentração de nitrito (Figura 1) é representado pela equação: Mortalidade $=-71,468+17,53 \times\left(\mathrm{mg} . \mathrm{L}^{-1} \mathrm{de} \mathrm{NO}_{2}\right)$, $\left(\left(R^{2}=0,62\right)\right.$, sendo que a concentração máxima utilizada, de $6,68 \mathrm{mg} . \mathrm{L}^{-1}$ de $\mathrm{NO}_{2}$, resultou uma mortalidade média de $45,63 \%$ dos animais em 96 horas.

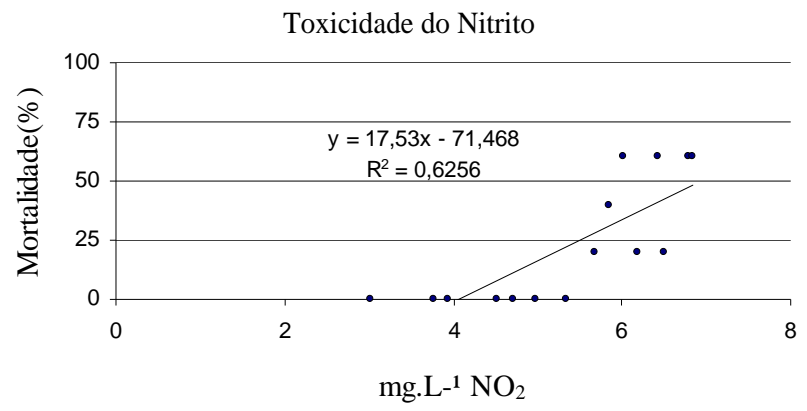

FIGURA 1 - Mortalidade de alevinos de cará em relação a concentração de $\mathrm{NO}_{2}$.

Este resultado é semelhante ao apresentado por Arana (1997), que afirma que a $\mathrm{CL}_{50}$ de nitrito em 96 horas para Channel catfish (Ictalurus punctatus), é de 7,6mg.L $\mathrm{L}^{-1}$ e, é muito inferior ao apresentado por Peres (2002), que apresenta uma $\mathrm{CL}_{50}-96 \mathrm{~h}$ de nitrito para larvas de Odontesthes argentinensis de $88 \mathrm{mg} \cdot \mathrm{L}^{-1}$.
Já Paula-Silva \& Pires-Lopes (1998) estudaram distúrbios no tecido sangüíneo de Colossoma macropomum resultantes da exposição a concentrações de 0 a $3,6 \mathrm{mg} . \mathrm{L}^{-1}$ de $\mathrm{NO}_{2}$, embora não tenham sido registradas mortalidades, foi observada alteração em 6 dos 10 parâmetros hematológicos avaliados, de forma que concentrações sub-letais de $\mathrm{NO}_{2}$ causam danos que podem se refletir nas funções fisiológicas básicas dos animais, crescimento e reprodução.

A concentração de amônia que causou a mortalidade de 50\% dos animais em 96 horas é representada (Figura 2) pela equação: Mortalidade $=-84,586+45,612 \times\left(\mathrm{mg} \cdot \mathrm{L}^{-1} \mathrm{de}\right.$ $\left.\mathrm{NH}_{3}-\mathrm{N}\right),\left(\mathrm{R}^{2}=0,59\right)$, resultando uma $\mathrm{CL}_{50}$, calculada de 2,95 mg. $\mathrm{L}^{-1}$ de $\mathrm{NH}_{3}-\mathrm{N}$

Estes resultados são muito superiores aos $0,80 \mathrm{mg} \cdot \mathrm{L}^{-}$ 'de $\mathrm{NH}_{3}-\mathrm{N}$, determinados por Ostrensky \& Brugger (1992), para alevinos de Odontesthes argentinensis e aos registrados por Sampaio \& Minillo (2000) também para larvas de Odontesthes argentinensis de 15 dias de idade, medindo $15 \mathrm{~mm}$ de comprimento e peso de $30 \mathrm{mg}$. Estes autores determinaram a $\mathrm{CL}_{50}$ entre $0,73 \mathrm{e} 0,96 \mathrm{mg} \cdot \mathrm{L}^{-1} \mathrm{NH}_{3}-$ $\mathrm{N}$, para temperaturas entre 15 e $23^{\circ} \mathrm{C}$ e salinidade de 28 , embora os mesmos alertem para o fato de haver consenso sobre o efeito da temperatura sobre a toxicidade da amônia em peixes. Por outro lado, o resultado obtido neste trabalho está na faixa de toxicidade sugerida por Abdalla \& 
MacNabb (1998) quando afirmam, que a concentração letal de amônia não ionizada para peixes varia entre 0,32 e 3,1 mg. $L^{-1}$ em exposição aguda de 96 horas, entretanto, o tamanho dos animais tem grande influência no nível de toxicidade, pois peixes menores recebem maior dosagem por unidade de peso do que peixes maiores, sendo, mais suscetíveis ao toxicidade da amônia não ionizada, o que explica a diferença entre os resultados dos diversos autores. Já, Cavero et al. (2004) expuseram juvenis de pirarucu a 2,0 mg.L. $\mathrm{L}^{-1}$ de $\mathrm{NH}_{3}-\mathrm{N}$ por 24 horas, e não observaram nenhum efeito no seu desempenho, atribuindo esta alta resistência à amônia pelo fato da espécie ter capacidade de respiração aérea, quando em condições adversas.

Toxicidade da Amônia

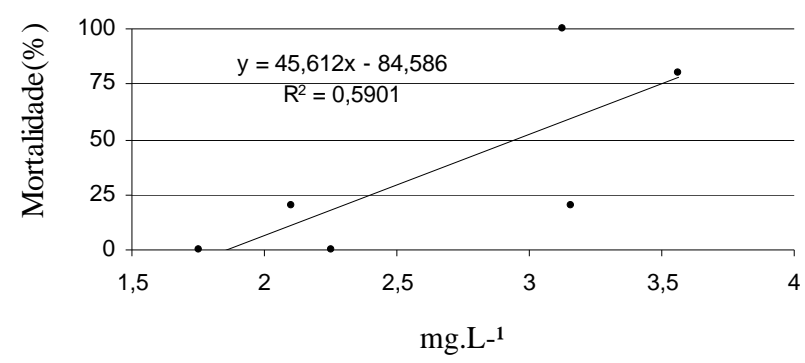

FIGURA 2 - Comportamento da mortalidade de alevinos de cará em relação a concentração de $\mathrm{NH}_{3}-\mathrm{N}$.

Referindo-se a presença da amônia não ionizada no ambiente, Bergmann (1994) alerta para o fato de que a toxicidade é resultado da sua interação com outros parâmetros, principalmente o $\mathrm{pH}$, a amônia ionizada e a atividade microbiana que ocorre no sedimento, podendo apresentar variações diuturnas de acordo com a intensidade dos processos de fotossíntese e respiração que ocorrem no ambiente, de modo que os resultados apresentados são relativos as condições experimentadas. Neste mesmo sentido, Spott (1979) afirma que trutas arco-íris (Salmo gairdneri) submetidas a elevados níveis de amônia ambiental, têm a amônia no sangue também aumentada, mas a concentração da amônia no sangue é sempre maior que a concentração de amônia ambiental. Concluindo, que um elevado nível de amônia na água causa uma retenção forçada da amônia metabólica a qual se torna tóxica.

Prova indireta dessa hipótese, segundo Spott (1979), foi obtida por Fromm \& Gillette (1968) quando demonstraram que a taxa de excreção de amônia na truta arco-íris decresce enquanto a concentração de amônia no ambiente sobe.
Para Evans (1993) os efeitos de uma elevada amônia total na água sobre a amônia excretada são similares àqueles do pH alto. Inicialmente, ocorre uma inibição da excreção de amônia e / ou entrada de amônia livre; em consequiência a amônia total no plasma aumenta. A entrada parece ser em grande parte devido à reversão do gradiente normal $\mathrm{P}_{\mathrm{NH}_{3}}$ do sangue para a água, o qual explica o bem conhecido efeito protetor do $\mathrm{pH}$ ambiental ácido sobre a toxicidade de amônia ambiental elevada.

A análise fatorial da variância da mortalidade em relação as concentrações de amônia não ionizada e de nitrito indicam haver interação entre os dois produtos (Pd" 0,05$)$, resultando aumento de mortalidade de alevinos.

Os resultados demonstram que a toxicidade do nitrito e/ou da amônia não ionizada, é muito específica tanto em nível de indivíduo, bem como de tamanho ou idade, sendo que os resultados aqui obtidos podem ser utilizados como referência de risco ambiental para o cará Cichlassoma facetum.

\section{CONCLUSÕES}

Concentrações na água, próximas aos 3,0 mg. $\mathrm{L}^{-1} \mathrm{de}$ amônia não ionizada e a 7,0 mg. $\mathrm{L}^{-1}$ de nitrito oferecem risco ambiental para populações de cará Cichlasoma facetum.

\section{REFERÊNCIAS BIBLIOGRAFICAS}

ABDALLA, A. A.; MACNABB, C. D. Acute and sublethal growth effects of um-ionized ammonia to nile tilapia Oreochromis niloticus. 1998. Disponível em: <http:// Lwww.hep.pac.dfo-mpo.gc.ca/congress 1998/nitrogen $>$. Acesso em: 8 jul. 2003 .

\section{AMERICAN PUBLIC HEALTHASSOCIATION. Standard methods for examination of water and wastewater. New York, 1998. 824 p.}

ARANA, L. V. Princípios químicos de qualidade de água em aqüicultura: uma revisão para peixes e camarões. Florianópolis: UFSC, 1997. 166 p.

BERGMANN, U. Chronic toxity of ammonia to the amphipod Hyalella azteca: importance of ammonium ion and water hardness. Environment Pollution, [S.1.], v. 86, p. 329-335, 1994.

CAVERO, B. A. S.; PEREIRA-FILHO, M.; BORDINHORI, A. M. Tolerância de juvenis de pirarucu ao aumento da concentração de amônia em ambiente confinado. Pesquisa Agropecuária Brasileira, Brasília, v. 39, n. 5, p. 513-516, 2004. 
CETESB. Água: teste de toxidade aguda com Daphnia similis Claus. 1876 (cladócera, crustácea). São Paulo, 1991. $33 \mathrm{p}$.

DUBOROW, R. M.; CROSBY, D. M.; BRUNSON, M. W. Ammonia in fish ponds. Southern Regional Aquaculture Center, [S.1.], n. 463, June 1997.

ESTEVES, F. A. de. Fundamentos de limnologia. Rio de Janeiro: Interciência, 1988. 575 p.

EVANS, D. H. The physiology of fishes. Boca Raton: CRC, $1993.590 \mathrm{p}$

FROMM, P. O.; GILLETTE, J. R. Effect of ambiental ammonia on blood ammonia and nitrogen excretion of rainbow trout (Salmo gairdneri). Comparative Biochmistry and Physiology, New Delhi, v. 11, p. 714-719, 1968.

GORSEL, M.; JENSEN, F. B. Nitrite uptake and HCO3 excretion in the intestine of the european flounder (Platichthys fleus). The Journal of Experimental Biology, Ottawa, n. 202, p. 2103-2110, 1999.

IBAMA. Manual de testes para a avaliação da ecotoxicidade de agentes químicos: teste para avaliação da mobilidade. Brasilia, DF, 1990.

KOCH, W. R.; MILANI, P. C.; GROSSER, K. M. Guia ilustrado de peixes do Parque Delta do Jacuí. Porto Alegre: Fundação Zoobotânica do Rio Grande do Sul, $2000.89 \mathrm{p}$.
OSTRENSKY, A.; BRUGGER, A. M. Studies on the viability of silverside Odontesthes argentinensis cultivations: acute toxicity of ammonia. Journal of the Brazilian Association for the Advancement of Science, [S.1.], v. 44, p. 413-414, 1992.

PAULA-SILVA, M. N. de; PIRES-LOPES, N. Nitrite causes hematological disturbances in Colossoma macropomum. 1998. Disponível em: $<$ http:///www.hep.pac.dfo-mpo.gc.cat congress 1998/nitrogens. Acesso em: 10 nov. 2004.

PERES, M. M. S. Toxicidade aguda e crônica de amônia e nitrito sobre larvas do peixe-rei marinho Odontesthes argentinensis. 2002. 26 f. Monografia (Graduação) Universidade Federal de Rio Grande, Rio Grande, 2002.

SAMPAIO, L. A.; MINILLO, A. Viabilidade do uso de larvas de peixe-rei Odontesthes argentinensis em testes de toxicidade: efeitos da salinidade e da temperatura sobre a toxicidade aguda da amônia. In. ESPINDOLA, E. L. G.; BOTTA-PASCHOAL, C. M. R.; ROCHA, O.; BOHRER, M. B. C.; OLIVEIRA-NETO, A. L. (Eds.). Ecotoxicologia: perspectivas para o século XXI. São Carlos: UFSCar, 2000. p. $545-553$.

SAS INSTITUTE. User's guide of Statistical Analysis System. 5. ed. North Carolina, 1998. 956 p.

SPOTT, S. Fish and invertebrate culture: water management in closed systems. New York: Willey, 1979. 177 p.

TIAGO, G. G. Aqüicultura, meio ambiente e legislação. Florianópolis: Annablume, 2000. 162 p. 